\title{
REMOTE METROLOGY, MAPPING, AND MOTION SENSING OF PLASMA FACING COMPONENTS USING FM COHERENT LASER RADAR *
}

\author{
M. M. Menon, ${ }^{1}$ R. E. Barry, ${ }^{1}$ A. Slotwinsky, ${ }^{2}$ H. W. Kugel, ${ }^{3}$ and C. H. Skinner ${ }^{3}$ \\ ${ }^{1}$ Oak Ridge National Laboratory \\ Robotics and Process Systems Division, \\ Oak Ridge, TN 37831-6305, USA \\ Telephone: 865-576-7505 \\ E-mail: menonmm@ornl.gov \\ ${ }^{2}$ MetricVision, 8500 Cinder Bed Road, Newington, VA 22122, USA \\ ${ }^{3}$ Princeton Plasma Physics Laboratory, Princeton, NJ 08543-0451, USA
}

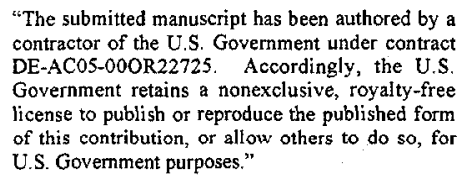

Oral Presentation, $21^{\text {st }}$ Symposium on Fusion Technology, September 11-15, 2000, Madrid, Spain. Proceedings of the conference will be published in the Fusion Engineering and Design journal.

* This work is supported by U. S. Department of Energy Contract DE-AC05-00OR22725 with the Oak Ridge National Laboratory, managed by UT-Battelle, LLC, and contract DE-AC02$76 \mathrm{CH} 03073$ with the Princeton Plasma Physics Laboratory, managed by Princeton University. 


\title{
REMOTE METROLOGY, MAPPING, AND MOTION SENSING
}

\section{OF PLASMA FACING COMPONENTS USING \\ FM COHERENT LASER RADAR}

\author{
M. M. Menon ${ }^{1}$, R. E. Barry ${ }^{1}$, A. Slotwinsky ${ }^{2}$, H. W. Kugel ${ }^{3}$, and C. H. Skinner ${ }^{3}$ \\ ${ }^{1}$ Oak Ridge National Laboratory, Oak Ridge, TN 37831-6305, USA \\ ${ }^{2}$ MetricVision, 8500 Cinder Bed Road, Newington, VA 22122, USA \\ ${ }^{3}$ Princeton Plasma Physics Laboratory, Princeton, NJ 08543-0451, USA \\ Corresponding author: M. M. Menon, tel. 865 - 576-7505, fax: $865-574-4624$ \\ e-mail: menonmm@ornl.gov
}

\begin{abstract}
Metrology inside a $\mathrm{D} / \mathrm{T}$ burning fusion reactor must necessarily be conducted remotely since the in-vessel environment would be highly radioactive due to neutron activation of the torus walls. A technique based on frequency modulated coherent laser radar (FM CLR) for such remote metrology is described. Since the FM CLR relies on frequency shift to measure distances, the results are largely insensitive to surface reflectance characteristics. Results of measurements in TFTR and NSTX fusion devices using a prototype FM CLR unit, capable of remotely measuring distances (range) up to $22 \mathrm{~m}$ with better than $0.1-\mathrm{mm}$ precision, are provided. These results illustrate that the FM CLR can be used for precision remote metrology as well as viewing. It is also shown that by conducting Doppler corrected range measurements using the CLR, the motion of objects
\end{abstract}


can be tracked. Thus, the FM CLR has the potential to remotely measure the motion of plasma facing components (PFCs) during plasma disruptions.

\section{OPERATIONAL PRINCIPLE}

The basic principle of operation of the CLR device is illustrated in Fig. 1. A laser beam, the frequency of which is linearly modulated in time, is focussed towards the target and the reflected beam is received using the same optical system. The range is determined by comparing the received signal with the reference signal, since the frequency shift is directly proportional to the round trip transit time of the beam. An array of precise range measurements on an object can be used to render a 3-D image of the object using special computer programs. Thus, the technique can be utilized for precision metrology as well as for viewing (inspection). The prototype CLR uses a class 1 diode laser $(<1 \mathrm{~mW}$ output) of frequency $193.5 \mathrm{THz}$ (1550 nm wavelength). The FM band is $100 \mathrm{GHz}$.

For a moving target, the Doppler contributions to range values from the up-sweep and the down-sweep regions (see Fig. 1) will be of equal magnitude but of opposing signs. Therefore, algebraic mean of the up-sweep and down-sweep range values gives the Doppler corrected range, while the difference between the up-sweep and the down-sweep ranges is proportional to the velocity of motion.

\section{MEASUREMENTS IN TFTR}

The ability of the FM CLR to conduct precision measurements in an environment involving high radiation and tritium is illustrated in this measurement. Flaking was observed on some of the graphite tiles covering the TFTR inner limiter. Measurements 
performed to elucidate the flaking problem included, tile alignment checks, scanning of different regions of the tiles, and estimating the size of a typical flake. Figure 2 shows a linear scan across two tiles, conducted to check the alignment. Good alignment is indicated between the tiles. Figure 3 was generated using range measurements performed on array of tiles. Figure 4 shows the results of a range scan made on a tile with two flaky regions. From this scan the area of the flake was estimated to be about $2 \mathrm{~mm} \times 3 \mathrm{~mm}$ and flake height was in the range of $0.3-0.6 \mathrm{~mm}$. Further details on the flaking phenomena of hydrogenated graphite tiles are published elsewhere [1].

\section{MEASUREMENTS IN NSTX}

A key feature in these measurements involved the technique of transferring the range measurements made from an arbitrary location of the FM CLR to the NSTX machine coordinates. The CLR was located at a convenient stable location (such as the base of the Bay K port). Targets (0.5000-inch spheres) were attached on reference monuments mounted on the vacuum vessel walls. The coordinates of these monuments in NSTX machine center-based coordinate system were known from earlier measurements made by a high precision $\left(10^{-4} \mathrm{inch}\right)$ commercial mechanical movable arm (MMA made by FARO). Range measurements were made on the reference targets from the CLR location. These measurements, together with the MMA calibration of these targets in the machine coordinate system, were used to transfer all subsequent range measurements from that particular CLR location to the machine coordinate system.

\subsection{Scan of the NSTX Plasma Facing Surfaces (PFCs)}


Figure 5 shows the range-based image of the plasma facing surfaces of the NSTX, including 5 toroidal line scans of the $\mathrm{ICH}$ antenna front surface. In constructing the image, the range measurements obtained from different ports were all transferred to the machine center based coordinate system. Such a scan can be used for alignment verification as well as for determining the precise position of the different plasma facing structures (e.g., the antenna surface) with respect to the machine axis and plasma.

\subsection{Examination of Fine Surface Features}

The NSTX center stack is covered with carbon-carbon composite tiles (Allied Signal 2-D CC Type 865-19-4) as well as sintered graphite tiles (Union Carbide ATJ). After the first cycle of plasma operation, the $\mathrm{C}-\mathrm{C}$ tiles showed tracks running in the toroidal direction, but the ATJ tiles were free from such track marks (see Fig. 6). A small area $\left(\sim 1.5 \mathrm{~cm}^{2}\right)$ of a tile with a track mark was scanned (Fig. 7) and the track depth was estimated to be about $0.3 \mathrm{~mm}$. The amount of carbon efflux into the plasma was estimated based on this measurement [2].

\section{MOTION DETECTION AND VELOCITY MEASUREMENTS}

To illustrate the ability of the FM CLR to track the motion of a moving object, range measurements were performed on the diaphragm of an audio speaker. Figure 8 shows the results. The Doppler corrected range values and the velocities of the diaphragm were determined from this measurement. This technique can be used to detect motion of certain plasma facing components during plasma disruptions. 


\section{ACKNOWLEDGEMENT}

This work is supported by U. S. Department of Energy contract DE-AC05-00OR22725 with the Oak Ridge National Laboratory, managed by UT-Battelle, LLC, and contract DE-AC02-76CH03073 with the Princeton Plasma Physics Laboratory, managed by Princeton University. 


\section{List of Figures}

Fig. 1 Illustration of the FM CLR technique

Fig. 2 Linear scan across two graphite tiles in TFTR

Fig. 3 Range based image of an array of graphite tiles

Fig. 4 Image of graphite tile with flaky regions.

Fig. 5 Range based image of PFCs in NSTX

Fig. 6 Photograph of a region showing tracking

Fig. 7 Frontal view of the region around the track (top) and side view showing the track depth (bottom).

Fig. 8 Deflection measurements using the CLR on a speaker diaphragm 

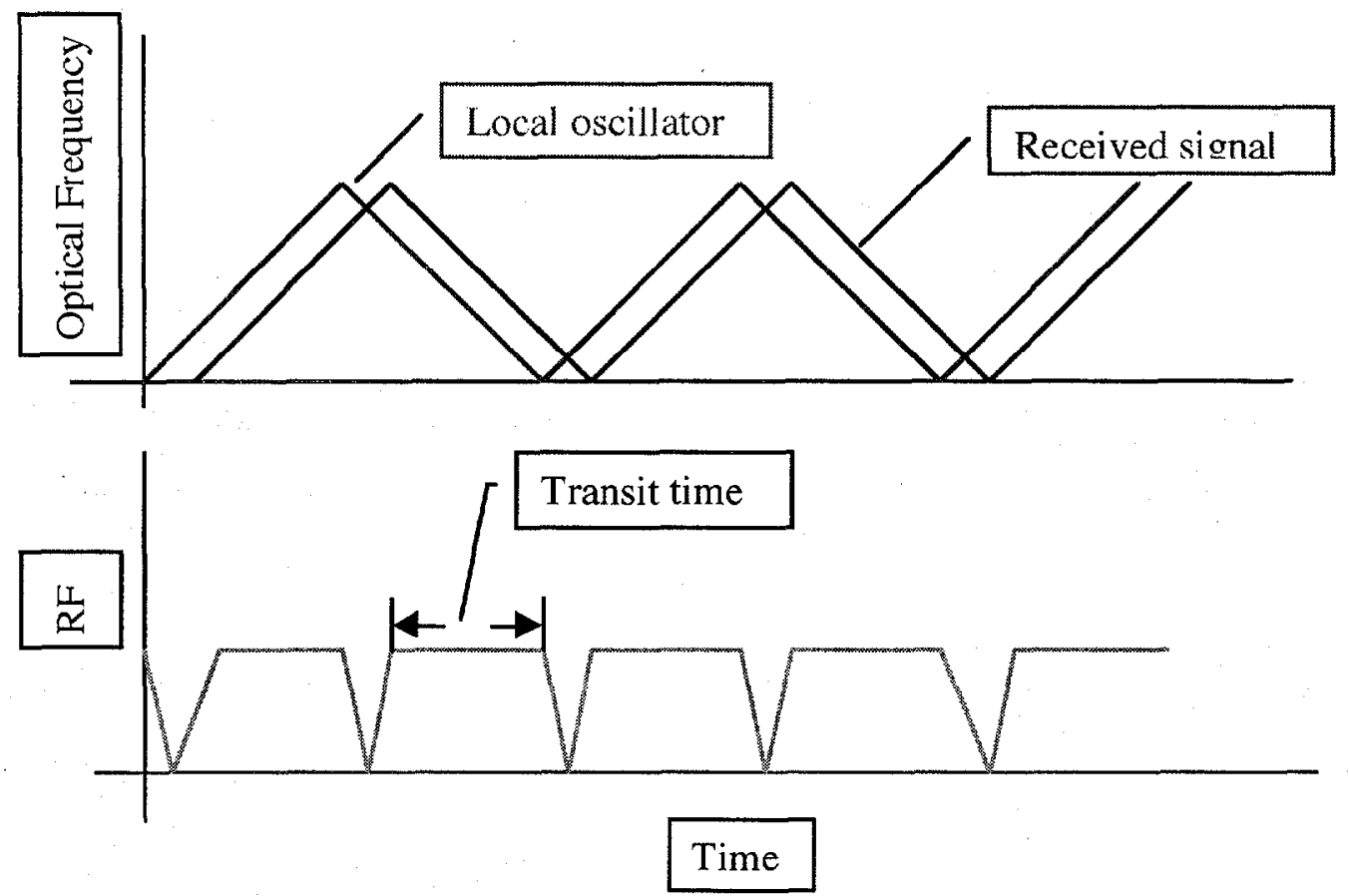


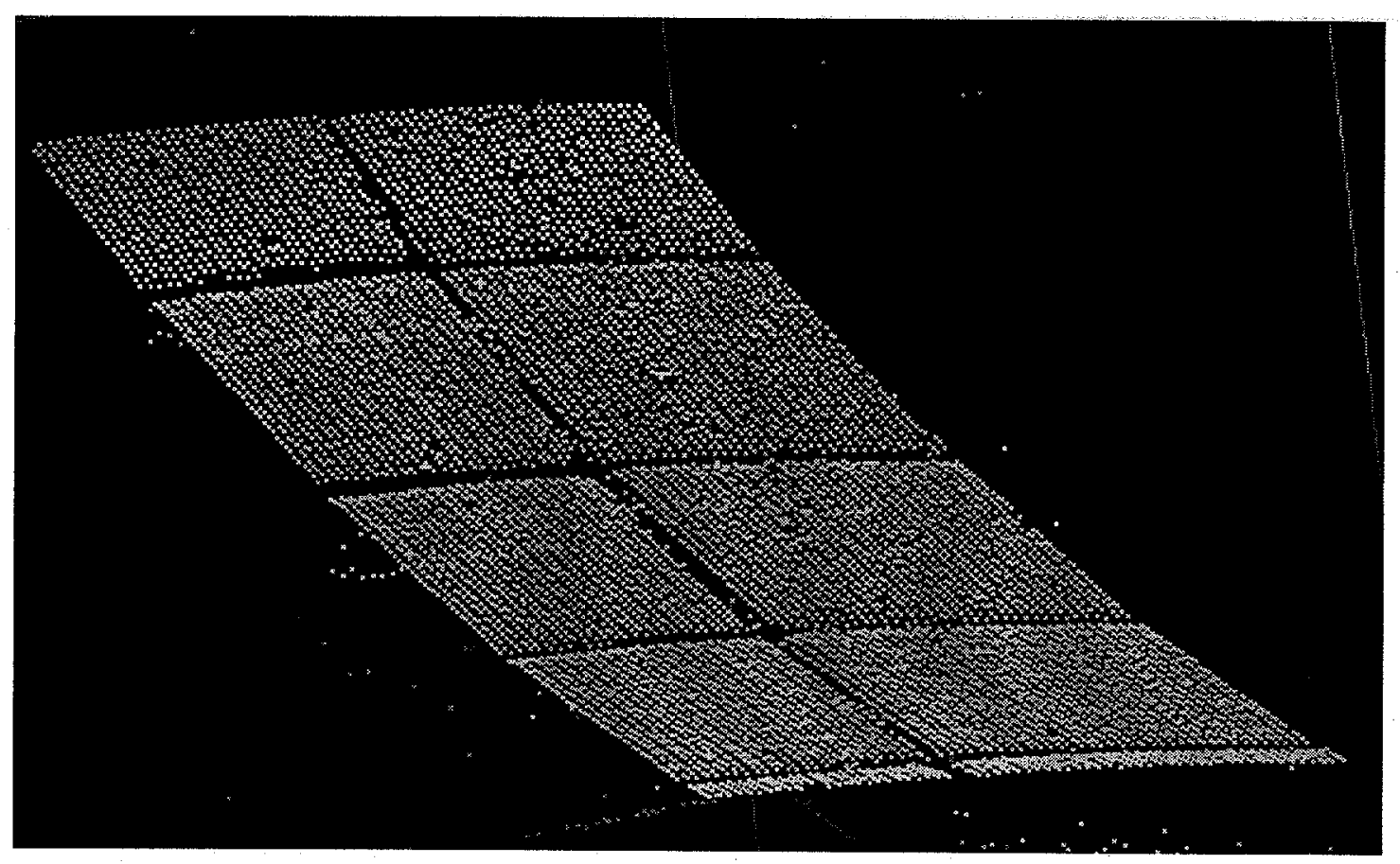




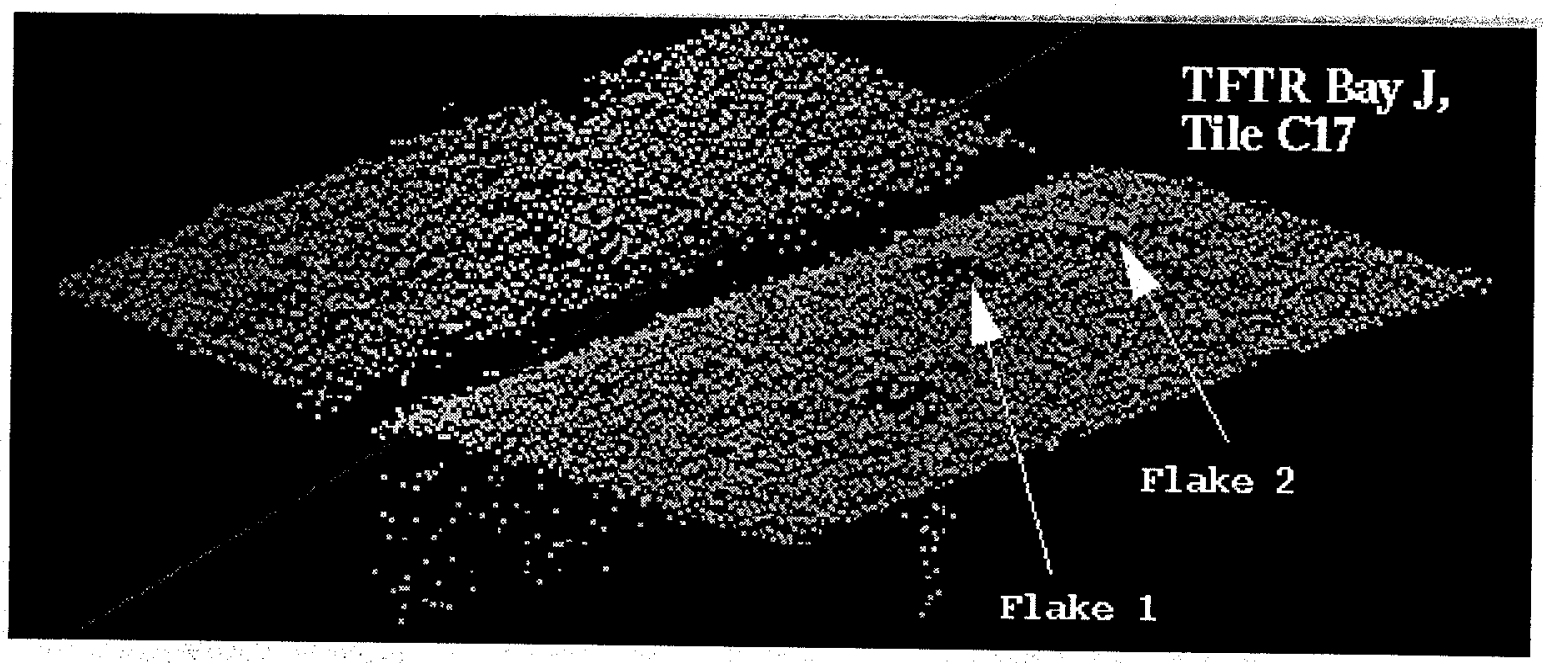




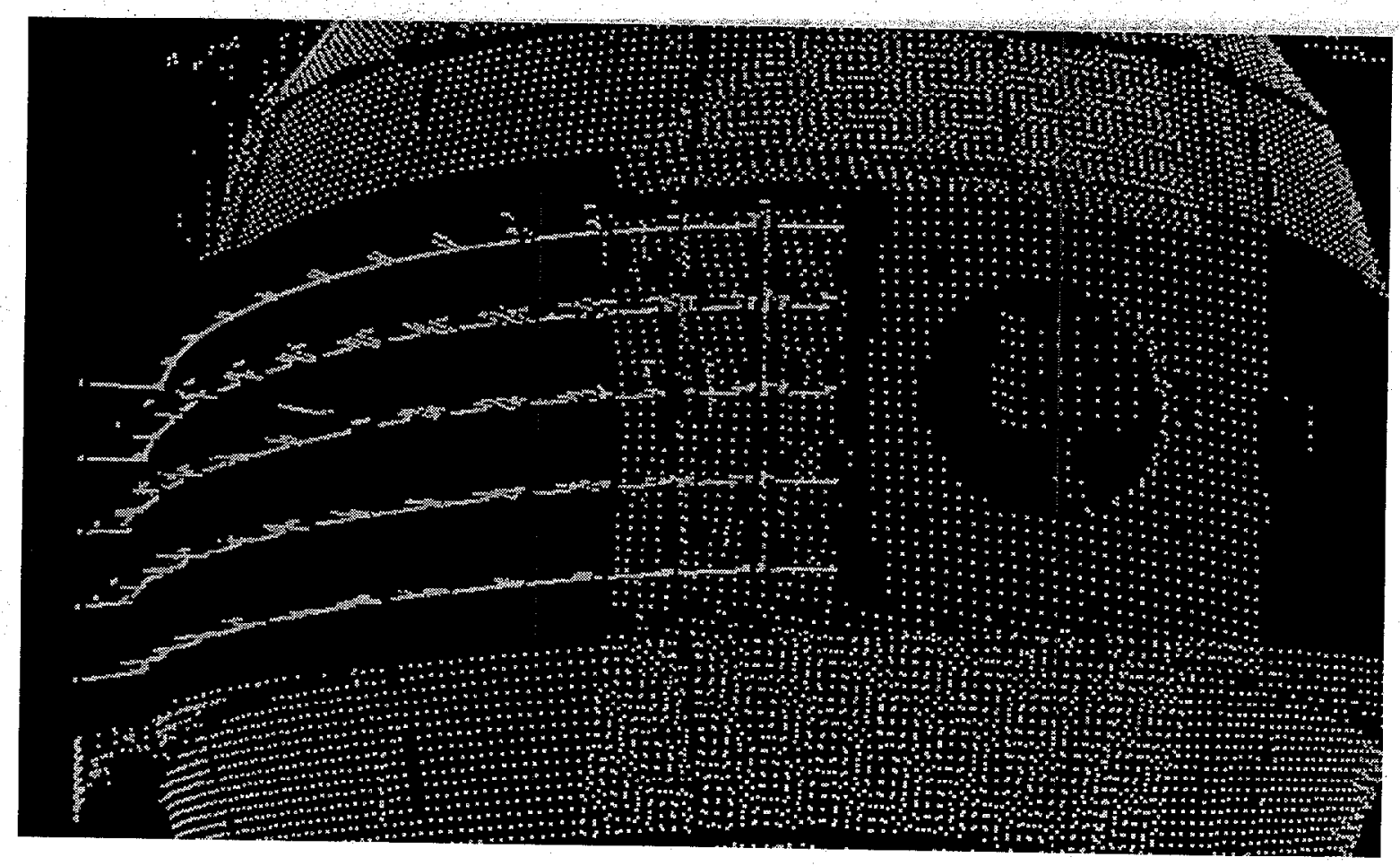




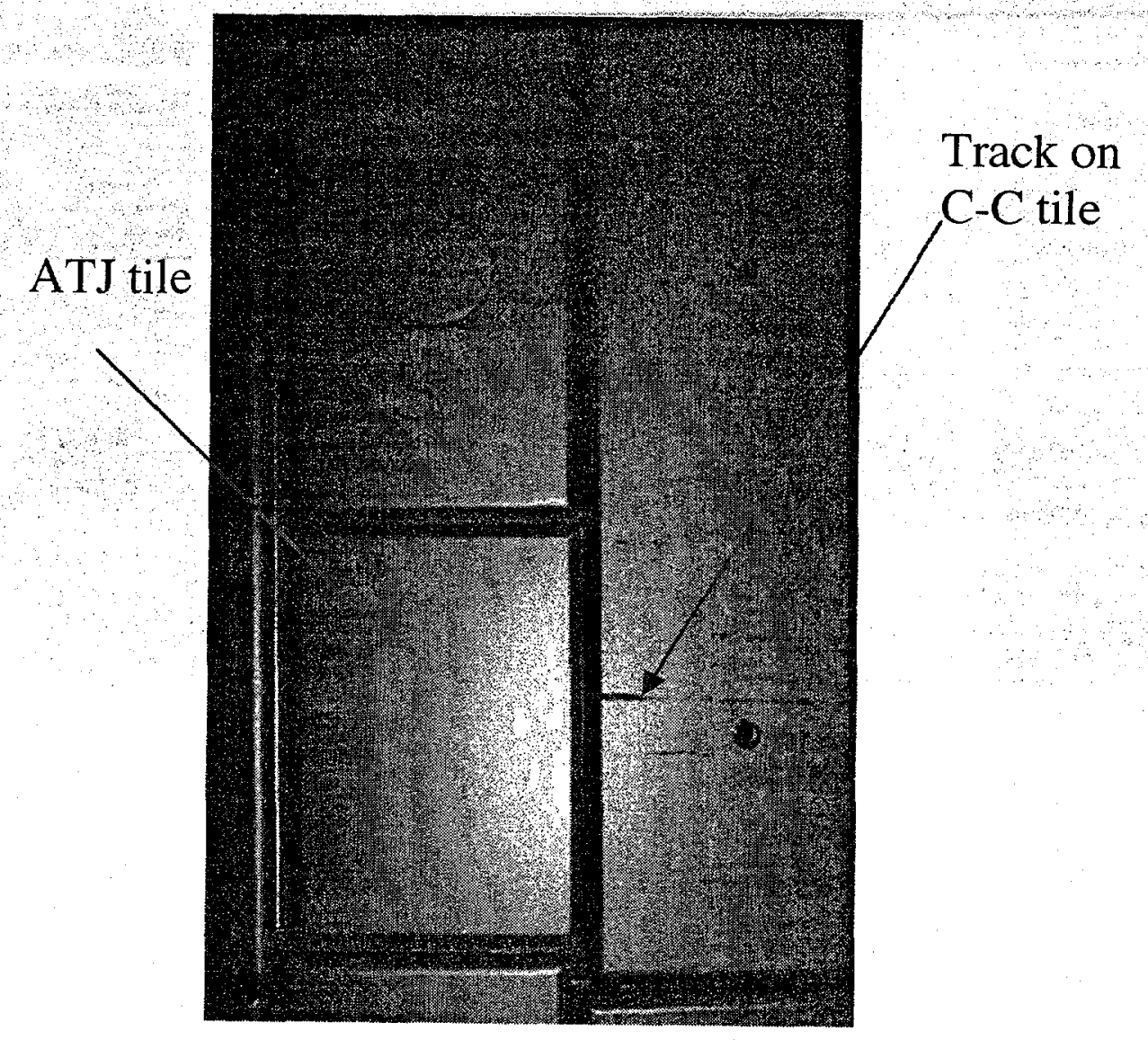



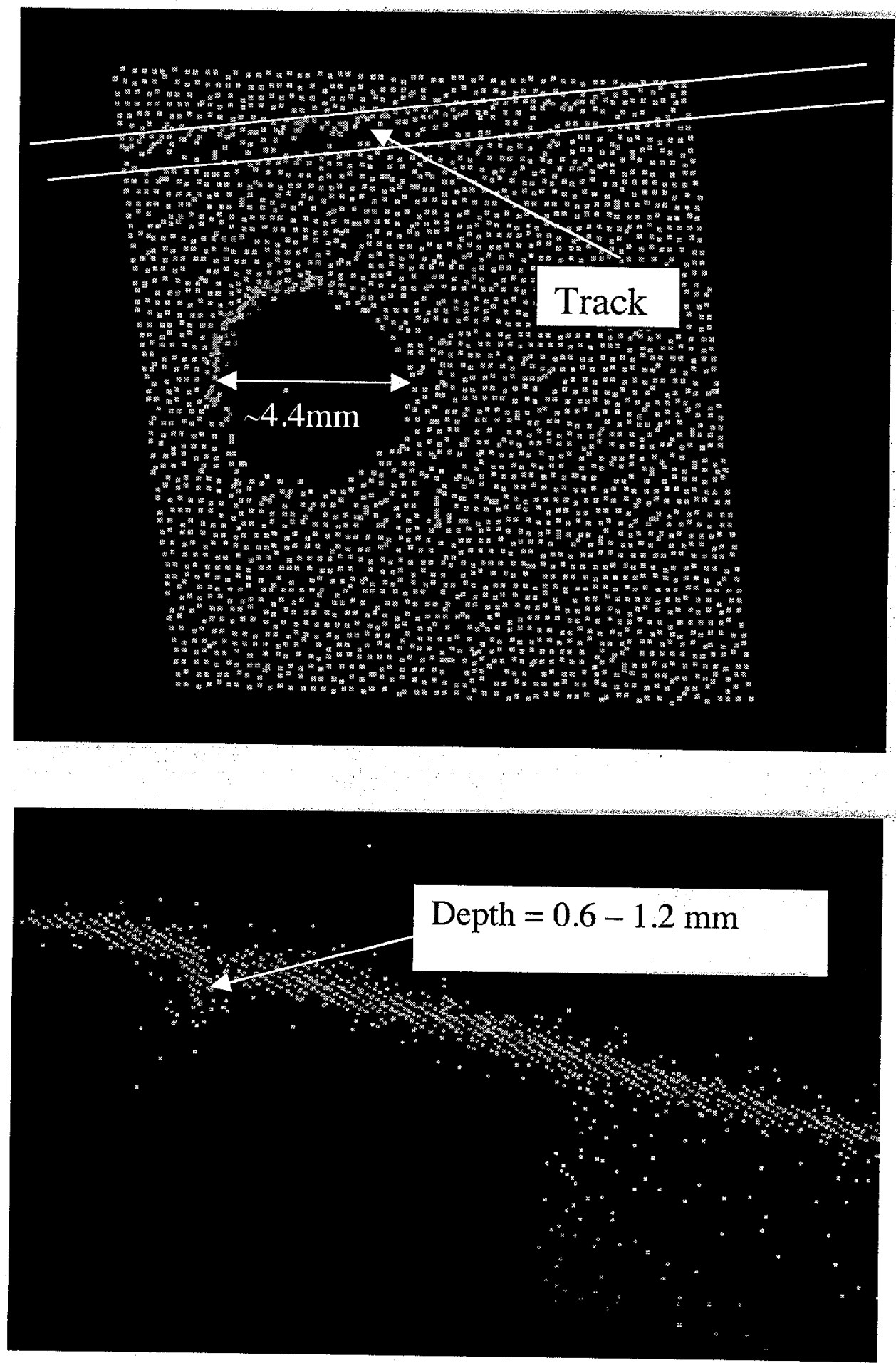


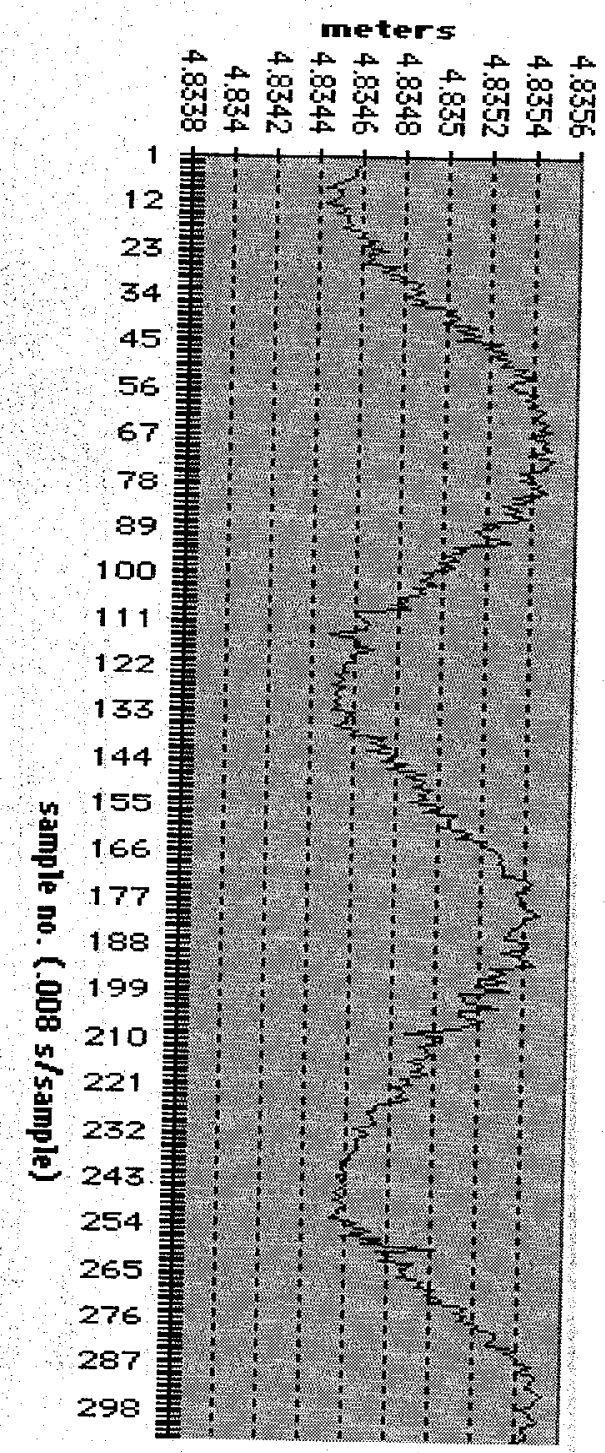

